\title{
Pseudodacryocystitis: paediatric case series of infected atypical ethmoid air cells masquerading as recurrent dacryocystitis
}

A Mohite ${ }^{1,3}$, T Jenyon ${ }^{1,3}$, B Manoj $^{2}$, S Sandramouli ${ }^{2}$, K Foster ${ }^{1}$, A Oates ${ }^{1}$, A-L McDermott ${ }^{1}$ and MV Parulekar ${ }^{1}$

\begin{abstract}
Purpose To highlight the clinical and surgical considerations in treating patients with apparent recurrent acute dacryocystitis with a patent lacrimal system.

Methods Three children referred to a tertiary unit as recurrent acute dacryocystitis were reviewed retrospectively. Imaging and subsequent surgical intervention revealed the underlying diagnosis.

Results All three cases presented with recurrent abscesses in the region of the lacrimal sac that failed to respond to incision and drainage. The lesions were

\section{Introduction}

Dacryocystitis usually presents secondary to nasolacrimal duct obstruction. Spread of inflammation from infected ethmoid air cells to the adjoining tissues can rarely mimic acute dacryocystitis in patients with a patent nasolacrimal system. ${ }^{1,2}$ We report three cases with clinically diagnosed recurrent dacryocystitis whose nasolacrimal system was shown to be patent. The underlying diagnosis was revealed to be infected low-lying anterior ethmoid air cells on computed tomography (CT) and magnetic resonance imaging (MRI). These were confirmed on subsequent surgical exploration.
\end{abstract} lower and more lateral to the usual location of a sac abscess and closer to the inferior orbital rim. All three cases were found to have patent lacrimal systems on syringing, and all were found to have infected, low-lying, anteriorly placed aberrant ethmoid air cells on computed tomography and magnetic resonance imaging. These were confirmed on subsequent surgical exploration.

Conclusions Infected low-lying ethmoid air cells can mimic dacryocystitis with recurrent abcesses. In cases where a patent nasolacrimal system is demonstrated and a more inferolateral location of the swelling than would be expected in dacryocystitis is seen, imaging is warranted to ensure the appropriate intervention is undertaken. Anterior ethmoidectomy as opposed to dacryocystorhinostomy is the appropriate treatment in these cases.

Eye (2017) 31, 657-660; doi:10.1038/eye.2016.282; published online 9 December 2016

\section{Case reports}

\section{Case 1}

LA, a 16-month-old girl, presented with suspected recurrent right-sided acute dacryocystitis with associated abscess. The abscess was initially treated with oral and topical antibiotics, and spontaneously ruptured, though did not fully resolve (Figures 1a and b). Subsequent examination under anaesthesia showed a patent nasolacrimal system on probing. The remaining cyst wall and surrounding granulation tissue were excised (Figure 1c). Rapid recurrence of three further abscesses occurred at same site, progressing to rupture within $48-72 \mathrm{~h}$. CT scans revealed the presence of infected, anteriorly placed aberrant ethmoid air cells as the origin of the abscess tract (Figures $1 \mathrm{~d}$ and e). Further incision and curettage, and removal of the mucosal lining by anterior ethmoidectomy at the site revealed a cavity extending to the bony orbital rim with no communication to the nasal cavity.
${ }^{1}$ Department of Ophthalmology, Birmingham Children's Hospital NHS Trust, Birmingham, UK

${ }^{2}$ Royal Wolverhampton NHS Trust, Wolverhampton, UK

Correspondence: M V Parulekar, Department of Ophthalmology, Birmingham Children's Hospital, Steelhouse Lane, Birmingham B4 6NH, UK Tel: +44 (0)12 1333 9477. Fax: +44 (0)1213339461 E-mail: manoj.parulekar@ bch.nhs.uk

${ }^{3}$ These authors contributed equally to this work.

Received: 29 August 2016 Accepted: 21 October 2016 Published online:

9 December 2016 


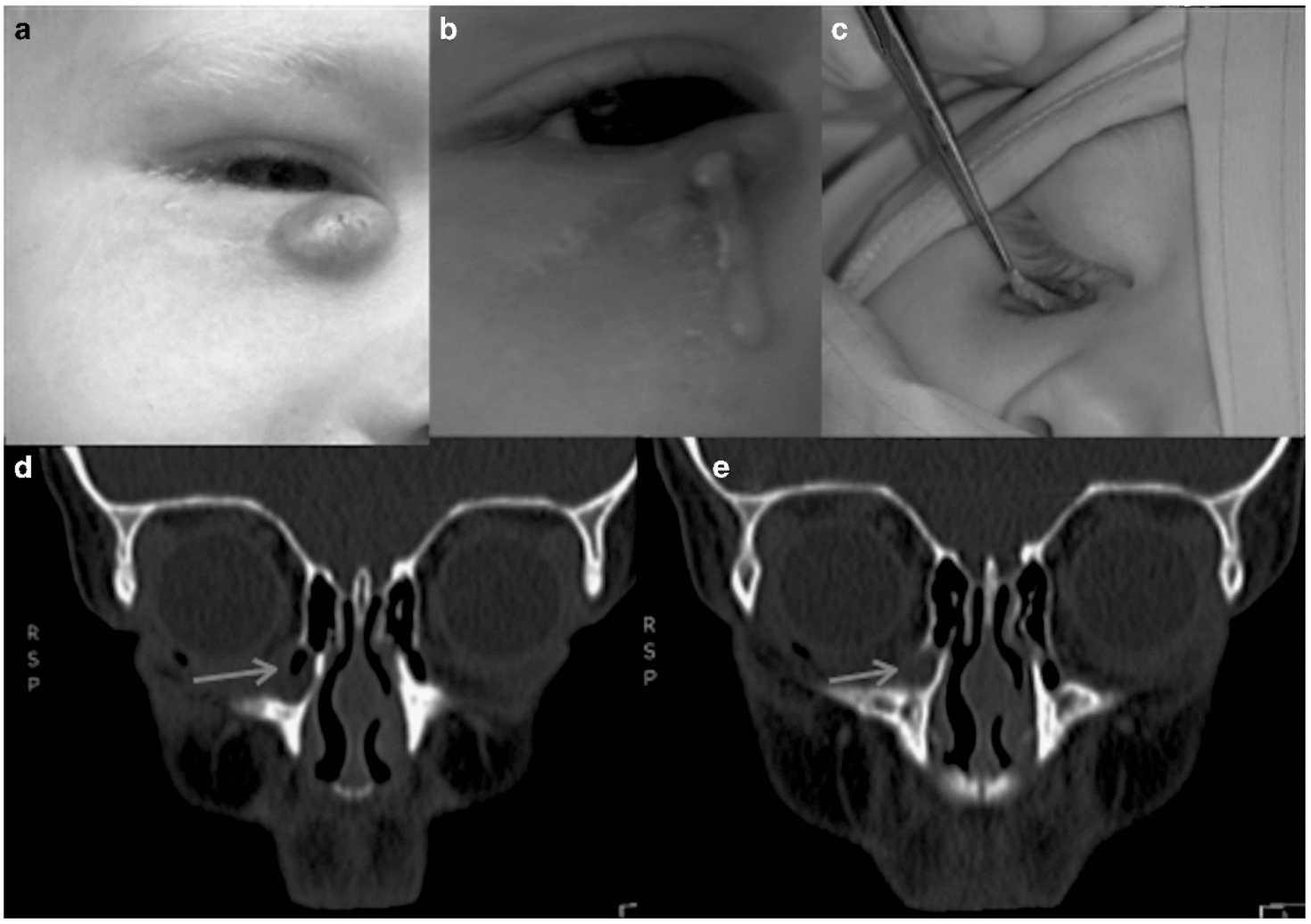

Figure 1 (a, b) Clinical progression of recurrent right lower lid swelling, with spontaneous rupture of abscess. (c) Excision of entire lesion sent for histology and microbiology. (d) Presence of ethmoid air cell corresponding to area of bony defeat at the bottom of abscess tract. (e) Corresponding air cell without any bony erosion suggesting infection came from the infected ethmoid air sinus.

\section{Case 2}

EW presented at 2 weeks of age with conjunctivitis. She subsequently developed an erythematous right lower lid swelling inferolateral to the sac region at 2 months of age and was treated with intravenous antibiotics for preseptal cellulitis. At 7 months of age, she was referred to us with the diagnosis of recurrent dacryocystitis (Figures 2a and b). Clinically, the abscess appeared lower on the lid than expected and the nasolacrimal duct was patent on probing. Incision and drainage was undertaken, but the abscess rapidly recurred. MRI head and CT scan of sinuses showed a collection adjoining the lacrimal sac (Figures 2d-f). Surgical exploration revealed a mucosallined collection, which was marsupialised, and monocanalicular intubation performed. Nasoendoscopic anterior ethmoidectomy was performed at the same time. This resulted in clinical improvement with no recurrence at 6 months follow-up (Figure 2c).

\section{Case 3}

EK presented at the age of four years with history of dacryocystitis with an associated recurring abscess inferolateral to the sac (Figure 3a). The abscess did not respond adequately to multiple courses of oral antibiotics and spontaneously discharged on one occasion. There was no history of epiphora, and syringing showed the nasolacrimal system to be patent. MRI and CT images showed opacification of the anterior, middle, and posterior ethmoid air cells together with inflammation spreading to the region of the nasolacrimal duct (Figures $3 b$ and $c$ ). The lacrimal system was patent, and an anterior ethmoidectomy was performed, resulting in complete resolution.

\section{Discussion}

Infected anteriorly placed ethmoid air cells can cause recurrent abscesses or extrinsic compression of the lacrimal system, and mimic dacryocystitis. A significant number of failed dacryocystorhinostomy (DCR) cases have been shown to have ethmoidal abnormalities suggesting that the occurrence of ethmoidal pathology mimicking or causing acute dacryocystitis may be common. ${ }^{3}$ In cases where a patent nasolacrimal system is demonstrated and a more inferolateral swelling is seen than would be expected in dacryocystitis, imaging is necessary to ensure the appropriate intervention is undertaken. Anterior ethmoidectomy as opposed to DCR is the appropriate treatment in these cases. 


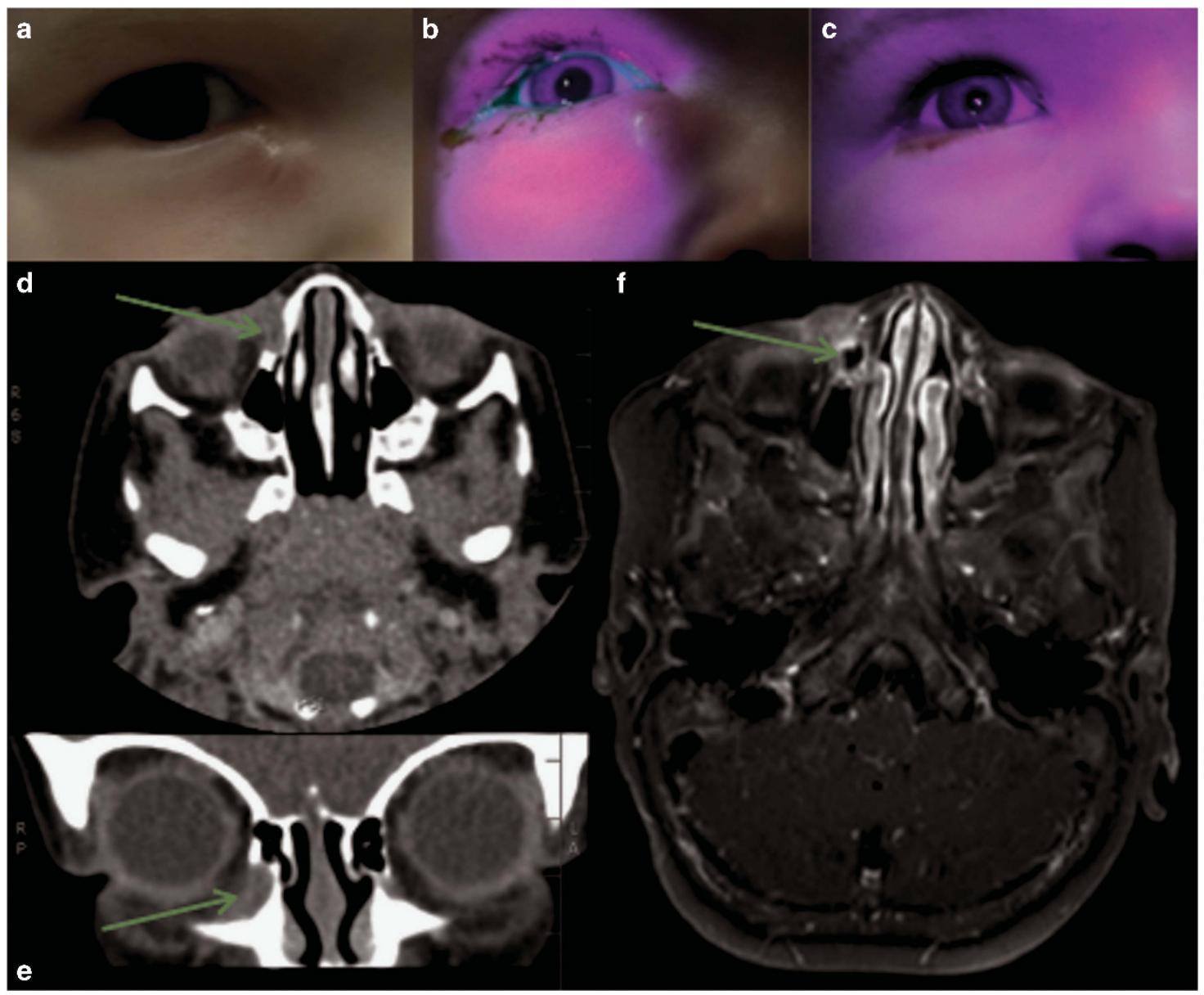

Figure 2 (a, b) Clinical photographs showing inflamed swelling inferior and lateral to right medial canthal tendon. Fluorescein pooling with acute inflammation. (c) Post-operative appearance following syringing and probing demonstrating a patent nasolacrimal system and anterior ethmoidectomy. (d, e) Axial and coronal CT images demonstrating abscess location. (f) T1-weighted axial MRI scan showing soft-tissue oedema with rim of fluid near medial canthal tendon, extending to inferior right meatus.

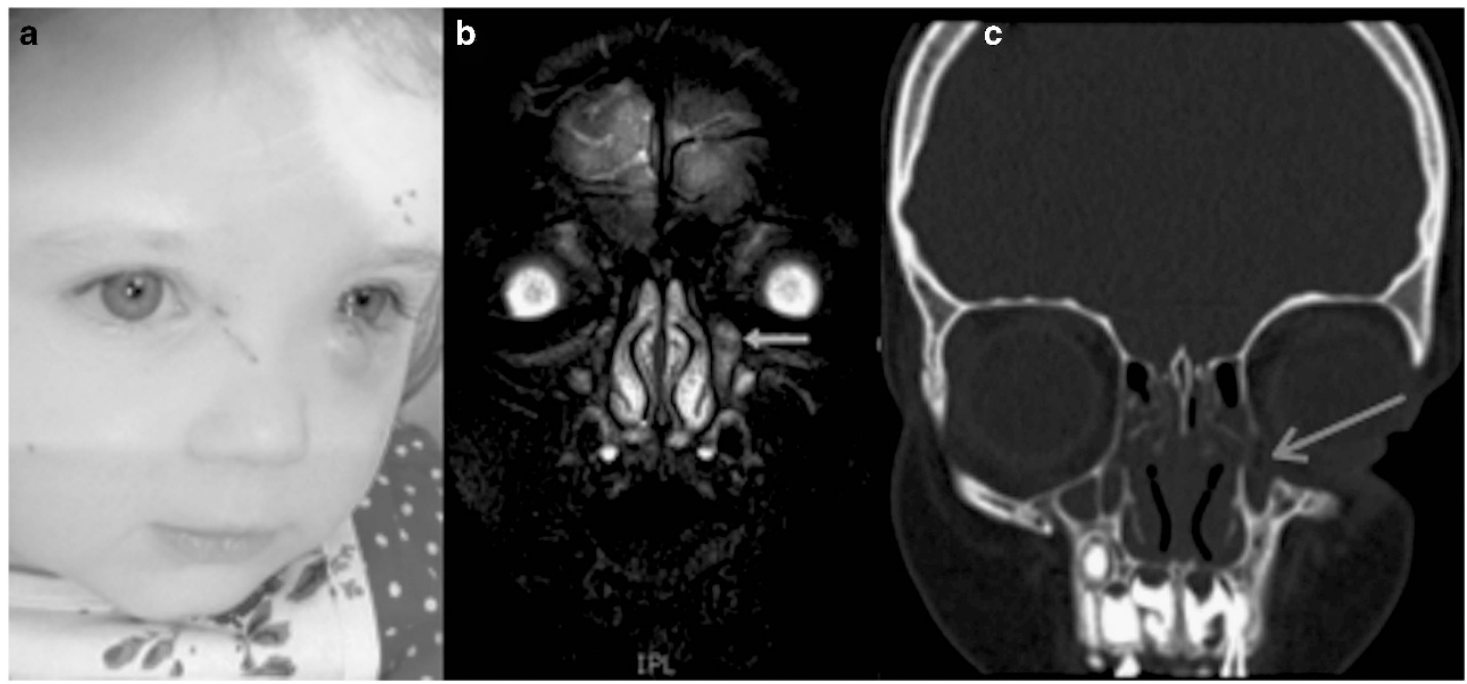

Figure 3 (a) Clinical photograph of left lower lid abscess. (b) Contrast-enhanced MRI scan showing mild expansion with associated increased enhancing soft tissue in the mid-distal portion of the left nasolacrimal duct. (c) CT scan of orbits showing extension of inflammation in the region of the nasolacrimal duct. 


\section{Summary}

What was known before

- Acute dacryocystitis usually occurs secondary to nasolacrimal duct obstruction.

- Symptoms include pain, redness, and swelling over the sac region, epiphora, and sometimes the development of an abscess.

- Management includes medical treatment with antibiotics, and surgical intervention with incision and drainage of abscess, or probing/dacryocystorhinostomy where indicated.

\section{What this study adds}

- In cases diagnosed as recurrent acute dacryocystitis, where a patent nasolacrimal duct is demonstrated, the underlying pathology may be an infected anteriorly placed ethmoid air cell.

- Clinical clues suggesting this include abscess formation more inferolateral to that which would be expected in dacryocystitis. In dacryocystitis, the swelling is directly below the medial canthal tendon.

- In such cases, further imaging is warranted (MRI/CT) to confirm the diagnosis and to guide surgical intervention (anterior ethmoidectomy).

\section{Conflict of interest}

This material is original research that has not been previously published and has not been submitted for publication elsewhere while under consideration. The authors declare no conflict of interest.

\section{Acknowledgements}

The study was supported by ophthalmology and radiology department in Birmingham Children's Hospital and ophthalmology department in The Royal Wolverhampton Hospital.

\section{References}

1 Yazici B, Yazici Z. Pseudodacryocystitis and nasolacrimal duct obstruction secondary to ethmoiditis. Ophthal Plast Reconstr Surg 2010; 26(5): 381-383.

2 Remulla HD, Rubin PA, Shore JW, Cunningham MJ. Pseudodacryocystitis arising from anterior ethmoiditis. Ophthal Plast Reconstr Surg 1995; 11(3): 165-168.

3 Liang J, Hur K, Merbs SL, Lane AP. Surgical and anatomic considerations in endoscopic revision of failed external dacryocystorhinostomy. Otolaryngol Head Neck Surg 2014; 150(5): 901-905. 